\title{
The neurologic consultation: pointers and takeaways for intensivists
}

\author{
Eelco F. M. Wijdicks ${ }^{*}$ (D) \\ (c) 2020 Springer-Verlag GmbH Germany, part of Springer Nature
}

Inevitably, critically ill patients in intensive care units exhibit neurologic manifestations [1]. Neurologic examination of the critically ill patient is complicated, but it is rarely taught, audited, or debated. Point-of-care diagnostic devices tempt over-reliance. The physiciandirected neurologic examination cannot become passé. When a patient changes neurologically, established principles inform and guide intensivists. The examination has its demands - ten of them, actually.

\section{History under pressure}

When pressed for time, a detailed history takes precedence over a detailed examination. We expect to miss important points in the history. Recounting circumstances immediately preceding or following the event falls mostly to laypersons. Answers may be evasive, even non-sequiturs. Overconfidence and framing bias lead to a bad start $[2,3]$. Taking a history is critical to a reliable neurologic diagnosis. A history of headache, earache, or backache suggests a previous spinal-cord abscess or meningitis in patients with septic shock. Prehospital cardiopulmonary resuscitation following traumatic head injury may change the prognostic equation. Use of contraceptives may indicate the hemorrhage is due to cerebral venous thrombosis Acute respiratory failure becomes neurologic if the history reveals dysphagia, muscle atrophy (motor neuron disease), fluctuating diplopia, jaw weakness, and exercise-induced or repetitive-use limb weakness (myasthenia gravis).

\section{Get off the scale!}

Scales summarizing neurologic examination are inherently insufficient. Few scales measure features needed

*Correspondence: wijde@mayo.edu

Division of Neurocritical Care and Hospital Neurology, Mayo Clinic, 200

First Street SW, Rochester, MN 55905, USA for urgent decisions. Scales are approximations and the basics of a neurologic examination (e.g., FOUR score in coma). Focal signs can be obvious (hemiparesis) or subtle (hemispatial neglect or anomic aphasia). "Top-down" neurologic examination includes language, executive function, brainstem reflexes, tone, strength, coordination, sensation, and reflexes (tendon, cutaneous, grasp, plantar, and coordinated nocifensive motor responses).

\section{Location, location!}

Neurology is, by definition, a localizing specialty. Therefore, once specific, focal findings appear, we must establish location (i.e., hemispheres, brainstem, or cerebellum-Figs. 1, 2). Not all regions represent eloquent parts of the brain; considerable injury may cause minor findings and vice versa. Findings not localized to specific structures may indicate global insults to the brain, often secondary to revived vital signs or abnormal physiology.

\section{Delirium: still in search of a name}

How should intensivists respond to delirious patients [4, 5]? Simply, find possible contributing or recently withdrawn drugs, query family members about substance abuse, and find a possible mimicking structural brain lesion or nonconvulsive status epilepticus in patients with "hypoactive delirium." Delirium may signify an unresolved (or new) systemic condition. Ask about cognitive history (patients with dementia frequently become delirious during hospital admission), intravenous drugs, hypotensive episodes, or a multi-day hypertensive surge. This suggests anoxic-ischemic brain injury, posterior reversible encephalopathy syndrome, or drug toxicityall presenting with acutely disturbed responsiveness.

\section{实



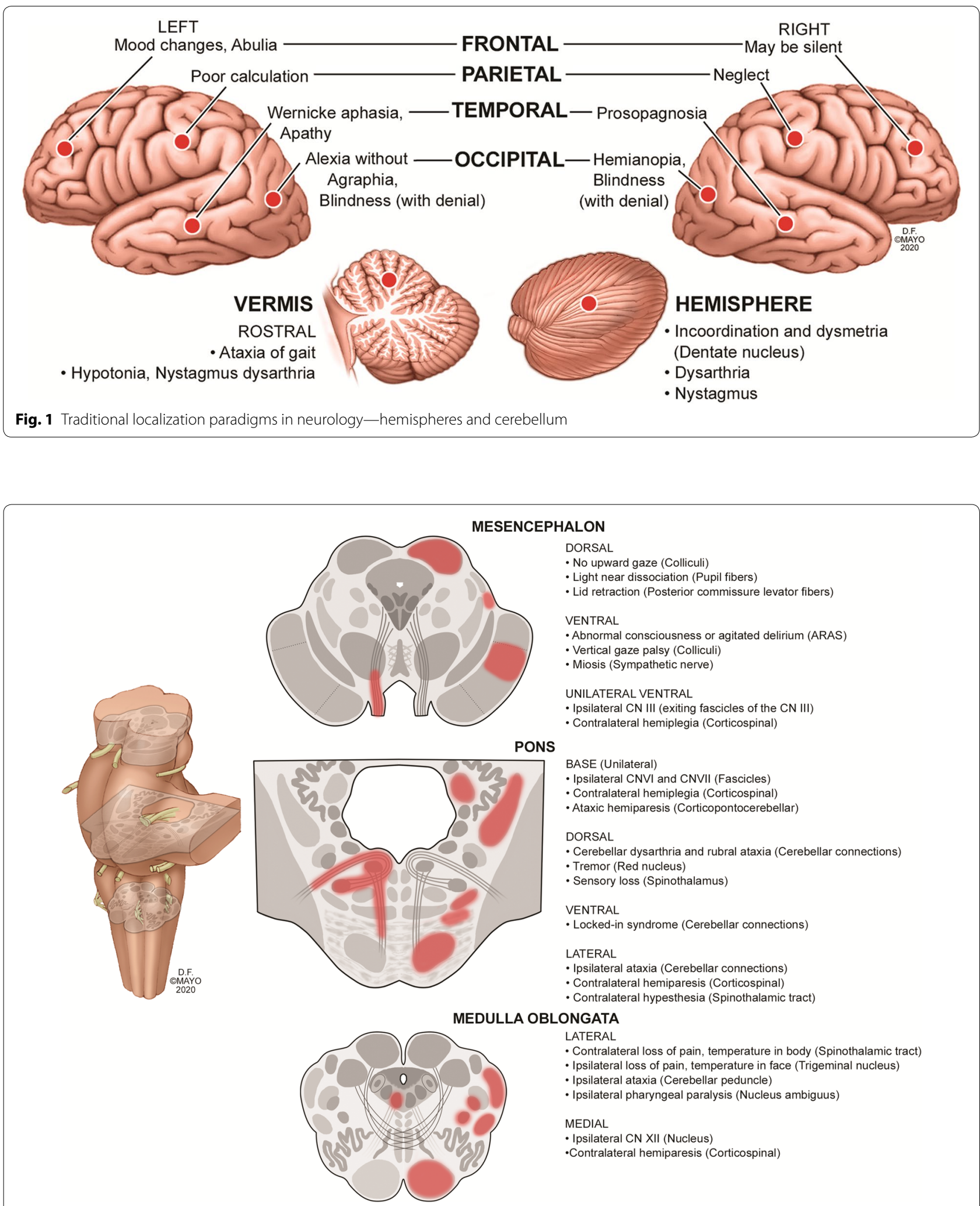

Fig. 2 Traditional localization paradigms in neurology—the brainstem 


\section{Sedation ruination}

Sedation is the bane of neurologists. Sedative-analgesic medications that treat distress in ventilated, critically ill patients confound neurologic examination and prognostication. Good judgment becomes impossible when recent intubation utilizes anesthetic drugs or neuromuscular blockers. Patients with prior IV infusions and organ dysfunction need time to wash these out. Physicians seldom account for these effects; recent evidence countermands these practices $[6,7]$.

\section{Unpredictive pupils}

Most critically ill patients have "normal" (possibly "sluggish") pupils [8]. Skilful examiners with magnifiers and a good light source may closely approximate pupilometers. Misplaced concerns about right- and left-sided differences increase unnecessary bedside calls. Anisoria is often irrelevant; nebulized drugs repeatedly cause widening pupils [9]. Often the only cranial nerves examined by non-specialists, pupils are insignificant outside five classic conditions with major implications: large and small pupil (acute basilar artery occlusion); unilateral wide, fixed pupil (acute brain shift); middle-position, fixed pupils (major upper-brainstem injury); or intoxication pupils, which widen with cocaine and constrict with heroin.

\section{Overlooked but should be looked over}

Eyes fixate to objects. With declining alertness, patients only open their eyes to prodding. As fixation tendencies disappear, eyes "rove" (i.e., move slowly, horizontally, and fitfully). Controlled only by subcortical circuits and the brainstem, eyes "bop," roll, "seesaw," and retract. Diffuse cortical or brainstem injury is likely with any of these movements, but none are specific or sensitive enough for prognostication. Dissociation between paired movements, due to one eye's failure to abduct or adduct (using ice-water tests), indicates brainstem involvement.

\section{Fascinating rhythms}

Prior seizure is often considered in unexplained coma; lateral tongue bites in patients with profound lactic acidosis clinch the diagnosis. It is more challenging with focal seizures, which are rhythmic, similar-amplitude contractions; all other movements are arrhythmic-shock-like (myoclonus), rigid posturing (dystonia and catatonia), or undulating (athetosis). Performed randomly with questionable indications ("altered mental status"), EEGs yield little-except over-consultation [10, 11]. Often, EEGs show nonspecific slowing or medication effects. Nonspecific triphasic waves or frontal, intermittent rhythmic delta activity are seen in patients with multi-organ failure. This pattern is most commonly misinterpreted as nonconvulsive status epilepticus.

There are two classes of movement abnormalities: hypokinetic disorders present with rigidity (and some myoclonus) and hyperkinetic disorders present as chorea, dystonia, tremor, or tics. Drowsy patients may display asterixis; stuporous patients may demonstrate widespread (multifocal) myoclonic jerks. Drugs causing oculogyric crises and oromandibular dyskinesis include phenothiazines and many antipsychotic drugs and, perhaps best known, metoclopramide. Acute movement disorders with specific treatment, such as malignant neuroleptic syndrome or serotonin syndrome, must be considered and cause severe rhabdomyolysis when there is rigidity and myoclonus $[12,13]$.

\section{There is no crystal ball}

Neurologists are often considered oracles, but they are not. They understand uncertainty and the dangers of extrapolation. ICU-generated prognostic data are multifactorial. We cannot just "plug in the numbers and crunch some data." There are few tipping points; the most crucial is secondary involvement of the upper brainstem.

\section{Trapped in limbo}

Why do we assume that unresponsive patients are unaware [14]? New technology detects responsiveness in coma patients who eventually awaken. New guidelines suggest late ( $>1$-year) improvements primarily occurring in younger patients and $\sim 20 \%$ of patients initially meeting criteria of a vegetative state [14]. Diagnostic error rates of $40 \%$ may be overstated due to insufficient neurologic skill and follow-up [15]. We do not know what to do here other than practice caution.

\section{Conclusion}

Neurologists have developed strategies to recognize a number of common, worrisome neurologic problems in critical illness. These takeaways are a typical representation of neurologic problems in any given situation.

\section{Acknowledgements \\ I thank David Factor for the medical illustrations. I thank Lea Dacy for deft editing}

Funding

No extramural funding supported this research.

Compliance with ethical standards

Conflicts of interest

None. 


\section{Publisher's Note}

Springer Nature remains neutral with regard to jurisdictional claims in published maps and institutional affiliations.

Received: 24 February 2020 Accepted: 11 April 2020

Published online: 24 April 2020

\section{References}

1. Wijdicks EFM (2016) Solving critical consults, 1st edn. Oxford University Press, New York

2. Saposnik G, Redelmeier D, Ruff CC, Tobler PN (2016) Cognitive biases associated with medical decisions: a systematic review. BMC Med Inform Decis Mak 16:138

3. Vickrey BG, Samuels MA, Ropper AH (2010) How neurologists think: A cognitive psychology perspective on missed diagnoses. Ann Neurol 67:425-433

4. Edlow BL, Chatelle C, Spencer CA et al (2017) Early detection of consciousness in patients with acute severe traumatic brain injury. Brain 140:2399-2414

5. Slooter AJC, Otte WM, Devlin JW et al (2020) Updated nomenclature of delirium and acute encephalopathy: statement of ten societies. Intensive Care Med. https://doi.org/10.1007/s00134-019-05907-4

6. Chanques G, Conseil M, Roger C et al (2017) Immediate interruption of sedation compared with usual sedation care in critically ill postoperative patients (SOS-Ventilation): a randomised, parallel-group clinical trial. Lancet Respir Med 5:795-805
7. Olsen HT, Nedergaard HK, Strom T et al (2020) Nonsedation or light sedation in critically III, mechanically ventilated patients. N Engl J Med 382:1103-1111

8. Oddo M, Sandroni C, Citerio G et al (2018) Quantitative versus standard pupillary light reflex for early prognostication in comatose cardiac arrest patients: an international prospective multicenter double-blinded study. Intensive Care Med 44:2102-2111

9. Braksick SA, Wijdicks EF (2014) Moisture and mydriasis. Pract Neurol 14:187-188

10. Kamel H, Betjemann JP, Navi BB et al (2013) Diagnostic yield of electroencephalography in the medical and surgical intensive care unit. Neurocrit Care 19:336-341

11. Kurtz P, Gaspard N, Wahl AS et al (2014) Continuous electroencephalography in a surgical intensive care unit. Intensive Care Med 40:228-234

12. Robottom BJ, Factor SA, Weiner WJ (2011) Movement disorders emergencies Part 2: hyperkinetic disorders. Arch Neurol 68:719-724

13. Robottom BJ, Weiner WJ, Factor SA (2011) Movement disorders emergencies. Part 1: Hypokinetic disorders. Arch Neurol 68:567-572

14. Giacino JT, Katz DI, Schiff ND et al (2018) Practice guideline update recommendations summary: disorders of consciousness: report of the guideline development, dissemination, and implementation subcommittee of the American Academy of Neurology; the American Congress of Rehabilitation Medicine; and the National Institute on Disability, Independent Living, and Rehabilitation Research. Neurology 91:450-460

15. Wade DT (2018) How often is the diagnosis of the permanent vegetative state incorrect? A review of the evidence. Eur J Neurol 25:619-625 